\title{
Colorectal Cancer Screening in a Spanish Town: Evaluation of Colonoscopy Preparation, Sedation by the Endoscopist and Screening Results
}

\author{
Rebeca Higuera Álvarez, Raquel González Monasterio, Fidencio Bao Pérez, \\ Pablo Ruiz Eguiluz
}

Gastroenterology Department, San Eloy Hospital, Bilbao, Spain

Email: rebecahiguera@yahoo.es

Received 31 March 2014; revised 5 May 2014; accepted 15 May 2014

Copyright (C) 2014 by authors and Scientific Research Publishing Inc.

This work is licensed under the Creative Commons Attribution International License (CC BY). http://creativecommons.org/licenses/by/4.0/

(c) (i) Open Access

\begin{abstract}
Objective: The aim of this study is to evaluate the colonic cleansing preparation and colorectal cancer screening results in Sestao (Bilbao); and to verify the safety of endoscopist-controlled sedation. Materials and methods: A retrospective observational study of colorectal cancer screening using the immunological faecal occult blood test in a medium-risk population in this region, with colonoscopy being performed in the event of a positive result. The colonic cleansing preparation was administered in split doses; the second dose was administered 4 hours prior to colonoscopy. The quality of the preparation and lesion detection were evaluated. Sedation-related complications were analyzed. Results: Participation was high (4342 of 6896 people invited, $62.99 \%$ ), with 309 subjects (7.12\%) presenting a positive immunological faecal occult blood test. A high percentage of the 284 colonoscopies had optimal preparation: $276(97.18 \%)$ had an adequate preparation. A total of $22(7.91 \%)$ sedation-related complications were detected: only one $(0.36 \%)$ was serious. A total of 140 high-risk adenomas were removed (47.13\%) and 24 carcinomas were diagnosed (8.08\%). Both these percentages are high. Conclusions: Split doses of colonoscopy preparation and reduction of the time interval between the second dose and the colonoscopy result in a high percentage of adequate preparation and lesion detection. Sedation by trained endoscopists is safe and effective. An immunological faecal occult blood test-based screening program is feasible and allows malignant and precancerous lesions to be efficiently detected and treated.
\end{abstract}

\section{Keywords}

Colorectal Cancer Screening Preparation Sedation 


\section{Introduction}

Colorectal cancer (CRC) is currently the most widely diagnosed cancer and the second leading cause of cancerrelated death in both the Western world and Spain when considering both sexes together [1] [2]. Its high incidence and mortality make this disease a public health problem. The natural history of CRC includes a preclinical phase which, in the sporadic phases that are the subject of screening, can last for up to 10 years (progression of an adenomatous polyp with low risk to high grade dysplasia and cancer). Detection of the disease during this phase improves its prognosis. CRC screening involves early detection and removal of premalignant adenomas or localised cancer, thus making treatment more effective and preventing cancer and cancer-related deaths. There is strong scientific evidence to show that CRC screening reduces CRC-related mortality [1]-[3].

Various CRC screening guidelines, such as the European guidelines, which recommend an annual or twoyearly faecal occult blood test (FOBT), rectosigmoidoscopy every five years or colonoscopy every 10 years in adults aged between 50 and 74 years with a medium risk of the disease but no other predisposing factors, have been published. The European Commission recommends that screening programs be undertaken in this medium-risk population as several clinical trials have demonstrated their efficacy, with a reduction in CRC incidence and mortality. A FOBT is the most common screening method [1].

The Spanish National Health System accepts this European recommendation to implement CRC screening programs, and is committed to achieving a population coverage of $50 \%$ by 2015 [4]. CRC screening in the medium-risk population of the Autonomous Community of the Basque Country (ACBC) was approved in May 2008 as a strategy for reducing CRC incidence and mortality. This project is currently in its final extension phase by region (phase III) to extend the current coverage from $71 \%$ to $100 \%$. The aim of this study is to evaluate the results of CRC screening in Sestao (Bilbao) and to analyse two strategies: improving the quality of the colonic cleansing preparation and to verifythe safety of endoscopist-controlled sedation.

Adequate colon preparation is vital for an effective colonoscopy that allows small polyps (less than $10 \mathrm{~mm}$ ) and flat lesions (higher risk of advanced neoplasia) to be detected, both of which are key to colorectal cancer screening programs, and to avoid the need for repeat colonoscopies due to inadequate preparation [5] [6].

Sedation has become an auxiliary technique that is required during numerous diagnostic and therapeutic endoscopic procedures, including screening colonoscopy, over the past few years, given that this technique is invasive, unpleasant and painful and often causes anxiety [7].

Adequate tolerance during a colonoscopy is also vital for a satisfactory examination.

The objective of this study is to evaluate the colonic cleansing preparation and colorectal cancer screening results in Sestao; and to verify the safety of endoscopist-controlled sedation.

\section{Materials and Methods}

We performed a retrospective observational study in the town of Sestao (Bilbao, Spain) (population: 28,959), which was part of the first phase of this CRC screening program in June-December 2011. The target population included 8069 men and women aged between 50 and 69 years (subjects with a personal history of CRC, a colonoscopy in the previous five years or digestive disease were excluded). The technique used for screening was a two-yearly immunological faecal occult blood test (iFOBT), which is a quantitative immunochemical method with a cut-off point of $\geq 100 \mathrm{ng} / \mathrm{mL}$ (positive) that only requires collection of one faecal sample, with no previous dietary restrictions, and can be completed at home after receiving the corresponding informative letter and kit. Samples were deposited at the ambulatory care centres. In the event of a positive iFOBT, a colonoscopy within a time interval of less than 30 days was requested via the primary care physician from a specific agenda at San Eloy Hospital.

The majority of colonoscopies were performed with endoscopist-controlled sedation and a different preparation than normally used, to administer a single dose more than 6 hours prior to the procedure. Thus, a three-day long low residue diet, followed by a first dose of magnesium citrate with 2 litres of fluid intake at $8 \mathrm{pm}$ the day prior to the procedure, followed by a second dose 4 hours prior to the procedure, was administered. No fluid ingestion was allowed two hours prior to the colonoscopy. This sequence was based on the recommendations in the American Society of Anesthesiologists (ASA) guidelines in order to prevent dehydration and bronchoaspiration [8].

The colonoscopy report contains a section for evaluating preparation quality using the modified Boston scale [9]: 1) Adequate: free from faecal matter or with few liquid remnants; 2) Acceptable: with a larger quantity of 
aspirable fluids or with few semi-liquid remnants that can be washed and aspirated; 3) Poor: semi-solid remnants that cannot be washed/aspirated or solid matter.

Regarding sedation, all subjects were provided with an informed consent form expressly stating that sedation would be performed by non-anaesthetist physicians (endoscopists trained and accredited by the Spanish Society of Digestive Endoscopy and Spanish Society of Critical, Intensive and Coronary Medicine Units) prior performing the examination.

Upon arrival at the endoscopy room, all subjects underwent a short clinical interview aimed at detecting problems that could pose a risk for the patient. This interview includes a series of questions using a template based on specific sedation questionnaires to determine comorbidity, ASA risk [10], drugs prescribed for the patient and the Mallampati score [11]. No pre-operative or anaesthesia consultation was held unless sedation was to be performed by an anaesthesiologist.

Sedation was performed by a team that included a physician, nurse and nursing auxiliary with endoscopic sedation and resuscitation knowledge, in a room suitably equipped with the materials required for advanced resuscitation according to Spanish Gastroenterology Association guidelines. A level IV or V sedation according to the Ramsay classification was performed in the majority of cases [10].

The drugs administered were midazolam, propofol at $1 \%$ dilution (as bolus and/or intravenous infusion) and/ or fentanyl.

Patients with ASA risk level III or IV, with active respiratory infection, complications during previous examinations with sedation or general anaesthesia or craniofacial malformations were sedated by an anaesthetist.

After the colonoscopy, patients were transferred to the Day Hospital, which was established in an adjoining room, for further observation and monitoring and for their recovery to be checked for as long as 30 minutes prior to discharge. Any complication observed during the examination was noted on the sedation form along with the drugs administered and measures taken. These aspects were also noted in the colonoscopy report.

The World Health Organization (WHO) criteria regarding histological polyp classification were applied, with any polyps encountered classified as high-risk adenomas (HRA) if $\geq 3$ adenomas or a size $\geq 10 \mathrm{~mm}$ or a vellous component or high-grade dysplasia in histology, including carcinomas in situ (Cis), were detected. Low-risk adenomas (LRAs) were defined as one or two adenomas of smaller size with a tubular histology and with a lowgrade dysplasia. Malignant polyps with carcinomatous transformation zones invading the submucosal layer (pT1), or invasive carcinomas, were classified as carcinomas [12] [13]. CRCs were classified according to the TNM system [14].

There is a fast internal follow-up loop (less than 30 days) involving Digestive, Radiology and Surgical Departments for cases of CRC and high-risk polyps. A report containing the specific recommendations (follow-up colonoscopy according to polyps removed, iFOBT after 10 years, exclusion from screening, etc.) is subsequently sent to the corresponding primary care physician.

The ACBC's CRC screening program has a unified information system that contains complete records for subsequent management, monitoring and evaluation at all health-care and public health levels organised by the program's Coordinating Centre. This application has a web that interoperates with other Osakidetza/Basque Health service applications with real-time data. The indicators used to evaluate the CRC screening program were: participation rate, cancer detection rate/1000 subjects screened, iFOBT results, colonoscopy results, positive predictive value (PPV) per 100 iFOBT-positives with colonoscopy examination, costs/organisational model.

Categorical and quantitative variables were expressed as absolute values or percentages and compared using Chi-squared. Results were expressed as prevalences with 95\% confidence intervals. Differences of $p<0.05$ were considered to be statistically significant. Statistical analysis was performed using the SPSS software package (version 20.0 for Windows) and WINPEPI (version 11.24).

\section{Results}

A total of 4342 (62.99\%) of the 6896 people invited decided to participate; 309 (7.12\%) tested FOBT-positive. Of these, 297 (96.12\%) agreed to undergo colonoscopy with sedation, whereas 13 (4.21\%) refused a colonoscopy. Four of the 297 subjects examined with colonoscopy presented inconclusive results (a colonic CT was performed in the event of incomplete colonoscopy to complete the study). The results for the remaining 293 subjects (98.65\%) were conclusive. A total of 13 subjects required sedation by an anaesthetist according to standard procedures (no fluid intake for at least 6 hours), whereas 284 were sedated by the endoscopist with a spe- 
cific preparation (completed 2 hours prior to the procedure). These latter subjects were analysed to assess preparation quality.

Subjects with an adequate preparation (259, 91.2\%) were considered to be those in whom the caecum and ascending colon could be correctly evaluated. A total of 17 colonoscopies (5.98\%) presented an acceptable preparation that could be improved to adequate by lavage and aspiration. A further eight colonoscopies $(2.82 \%)$ exhibited poor presentation and had to be repeated.

Preparation in the ACBC screening (conventional preparation method) was good in $85.7 \%$ of cases, acceptable in $8.2 \%$ and poor in $6.1 \%$.

Regarding sedation, four of the 297 colonoscopies performed as part of the screening program could not be analysed due to lack of information in the sedation section, a further two subjects underwent the procedure without sedation and 13 were sedated by an anaesthetist. A total of 278 colonoscopies were performed with endoscopist-controlled sedation. Of these, 22 (7.91\%) presented complications, and only one $(0.36 \%)$ was serious (apnoea). The latter required the examination to be suspended and a longer than usual observation time, although the patient recovered fully. Seventeen of these complications occurred in men and five in women, with seventeen presenting ASA II and five ASA I.

The complications observed were as follows: nine cases of bradycardia (3.24\%), four of mild desaturation (1.44\%), four of hypotension (1.44\%), two of hypertension ( $0.72 \%)$, one of severe desaturation $(0.36 \%)$, one of apnoea $(0.36 \%)$ and one of skin rash $(0.36 \%)$.

As the preparation was taken 2 hours prior to colonoscopy with sedation, no cases of bronchoaspiration were observed.

The CRC screening results for Sestao can be found in Table 1. A total of 35 LRAs (12.11\%) and 10 nonadenomatous polyps (3.46\%) were found. Seven of the 24 carcinomas detected presented in an advanced stage (III and IV, Dukes C and D; 29.17\%). There were no significant differences regarding participation and CRC distribution by age range or sex in this screening region.

In the first round of the screening program for the ACBC as a whole (2009 - 2011), 230,505 out of a total of 547,000 people were invited. The results of this round can also be found in Table 1. A higher number of lesions (LRA, HRA and CRC) were found in men. The PPV (predictive positive value) for iFOBT in CRC (8.8\%, $p=$ 0.012) was higher for the 65 - 69 years age group.

As reflected in Table 2, cancer detection per 1000 subjects screened and HRA rates in Sestao are higher than in the rest of the ACBC and Spain as a whole.

\section{Discussion}

The results of our study show a high percentage of participation for iFOBT-based CRC screening programmes, with a higher quality colonoscopy preparation, mainly due to the shorter interval between administration of the second dose and the procedure. A higher percentage of premalignant lesions and cancer was/were also detected. We have also shown that endoscopist-controlled sedation is both safe and effective.

European guidelines recommend participation rates greater than $60 \%$ in population-based CRC screening programs to ensure their cost effectiveness [1]. The percentage participation in this study (63\%) is high in comparison with screening programs undertaken in other Autonomous Communities in Spain, and with comparative population-based screening studies (less than 45\%) [15]-[17]. Involvement of the Primary Care sector and the ease with which the iFOBT is performed may have influenced these results.

The early detection of advanced adenomas is the key to CRC prevention since these lesions are more likely to progress to cancer. However, adequate cleansing of the colon is required to be able to detect these lesions, especially polyps with a size less than $9 \mathrm{~mm}$ and flat lesions. This is important because approximately half of all surface carcinomas detected derive from flat lesions [6]. The number of polyps detected (which should be more over $20 \%$ after iFOBT-based screening), removed (all smaller than $2 \mathrm{~cm}$ ) and their recovery (more over $80 \%$ of those $<10 \mathrm{~mm}$ and more over $95 \%$ of those $>10 \mathrm{~mm}$ ) are all colonoscopy quality indicators. All of these indicators are met in our screening. Indeed, a higher number of polyps analysed indicate a better visualisation and better preparation of the mucosa. A high-quality colonoscopy is vital for the success of any screening program [3] [18]. The fact that a higher than normal percentage of polyps and cancer were detected in this study could be due to the high percentage of participation and the number of iFOBT-positive results. Previous studies in this respect have shown that an iFOBT is more effective than FOBT with guaiac for detecting CRC and advanced adenomas 
Table 1. Overall CRC screening results for Sestao compared to those of the ACBC as a whole (participation, iFOBT and lesion detection).

\begin{tabular}{|c|c|c|c|c|c|c|c|c|}
\hline & \multicolumn{4}{|c|}{ SESTAO } & \multicolumn{4}{|c|}{$\mathrm{ACBC}^{1}$} \\
\hline & $\mathrm{N}$ & $\%$ & $95 \%$ CI & $\mathrm{VPP}^{2}$ & $\mathrm{~N}$ & $\%$ & $95 \%$ CI & $\mathrm{VPP}^{2}$ \\
\hline Invited & 6.896 & & & & 230.505 & & & \\
\hline Participation & 4.342 & 62.99 & $61.8-64.1$ & & 148.249 & 64.3 & $64.1-64.5$ & \\
\hline iFOBT $^{3}$ & 309 & 7.12 & $6.4-7.9$ & & 9.933 & 6.7 & $6.6-6.8$ & \\
\hline Colonoscopy & 297 & 96.12 & $93.3-98$ & & 9.248 & 93.1 & 92.6 - 93.6 & \\
\hline HRA $^{4}$ & 140 & 47.14 & $41.35-52.99$ & 47.14 & 3.952 & 42.6 & $41.6-43.61$ & 42.6 \\
\hline $\mathrm{CRC}^{5}$ & 24 & 8.08 & $5.24-11.79$ & 8.08 & 571 & 6.2 & $5.7-6.7$ & 6.2 \\
\hline
\end{tabular}

${ }^{1}$ Autonomous Community of the Basque Country; ${ }^{2}$ Positive predictive value (PPV) per 100 iFOBT-positives with a colonoscopic examination; $p<$ $0.05 ;{ }^{3}$ Immunological faecal occult blood test; ${ }^{4}$ High-risk adenoma; ${ }^{5}$ Colorectal cancer.

Table 2. Cancer detection and HRA rates/1000 persons screened.

\begin{tabular}{cccc} 
& SESTAO & ACBC $^{1}$ & NHS $^{2}$ \\
\hline HRA $^{3}$ & 37.77 & 26.3 & 20.55 \\
CRC $^{4}$ & 5.52 & 3.8 & 2.97 \\
\hline
\end{tabular}

${ }^{1}$ Autonomous Community of the Basque Country; ${ }^{2}$ National Health System; ${ }^{3}$ High-risk adenoma; ${ }^{4}$ Colorectal cancer.

[13] [19]. As this latter test has been used in other Autonomous Communities, this may partially explain the higher detection of high-risk lesions in the ACBC. However, when analysing the results, a bias in the ACBC CRC screening program resulting from the fact that subjects with a family history of CRC were not systematically excluded must be taken into account. A higher risk of CRC diagnosis with a family history of CRC has been observed in another CRC screening [20]. Despite this, the detection rate for premalignant and cancerous lesions in Sestao remains higher than the rates for the rest of the ACBC. As a result, we must consider whether there may be a higher prevalence of CRC in this region. In light of this, we analysed the age range to determine whether it had any effect on the higher lesion detection rate as a greater number of lesions are detected in the range 60 - 69 years [17] [21]. However, the mean age for all patients with lesions was 60 years and the number of patients aged 60 - 69 years was not sufficiently high to explain our findings. One of the factors that are likely to explain this higher lesion detection rate is the better colonic preparation procedure used compared to the conventional procedure which is used in the rest of the region.

The effect of preparation on colonoscopy is important due to the fact that preparation is sub-optimal in as many as $20 \%$ - $25 \%$ of cases, with only $20 \%$ of these being due to poor compliance. Other independent factors, such as prior surgery, advanced age, male sex, dementia, stroke and cirrhosis, also result in poor preparation. Factors that can be corrected, such as intolerance, drugs that slow intestinal rhythm or the time at which the preparation is administered, also have an effect [22]. Poor preparation results in a lower caecal-intubation and polyp-detection rate, thereby increasing the interval cancer risk and the risks and costs arising due to prolonged and repeated examinations [23].

The adequate preparation percentages described for this series are higher than those found using the conventional preparation ( $97.18 \%$ vs. $82 \%$ - 86\% respectively). Several reviews and studies have shown differences in colonic cleansing efficacy depending on the preparation administration time [6] [24].

One of the quality criteria used in the field of colonoscopy is to achieve a good or very good preparation in more than $95 \%$ of cases [23]: more than $90 \%$ is acceptable, but more than $95 \%$ is desirable [1]. A value of $97.18 \%$ was obtained in our study.

It has been shown that the quality of the preparation is higher if it is administered in various doses and that a reduction in the time interval between administration of the second dose and the start of the colonoscopy to 4 hours is the most influential factor [6] [25]-[27]. 
The consequences of a better preparation quality for the colonoscopy are a higher percentage of caecal intubations with correct evaluation of the caecum and ascending colon (which is where most faecal remnants are found if the preparation is not adequate) and therefore a greater possibility of detecting flat lesions and polyps [18], a lower colonoscopy repetition rate, lower risk and lower cost.

The preparation was administered closer to the examination and, in accordance with the indications of the ASA guideline, with no fluid intake for at least two hours, thereby reducing the risk of dehydration. Furthermore, as confirmed in our study, this minimum time essentially eliminates the risk of bronchoaspiration in healthy adults [8].

Sedation by non-anaesthetist physicians is common in daily clinical practice, maintaining an optimal level of safety by selecting patients on the basis of their ASA risk. Indeed, the low adverse event rate highlights the safety of sedation by non-anaesthetist physicians. Propofol is one of the most widely used drugs in endoscopic sedation due to its fast action and short half-life. Sedation induction is lower with propofol than with benzodiazepines and there is no difference in the procedures undertaken in patients sedated with propofol or with a narcotic and midazolam [28]. Furthermore, a short medical history aimed at detecting problems that may pose a risk to the patient must be taken. Moreover, various tools for selecting the sedation technique, such as the ASA classification, which classifies patients in a fast and easy manner and is widely accepted and used, and the Mallampati index, which can be used to evaluate the risk of a difficult intubation and predicts ventilation problems, are available [11]. The drug administered for sedation, and its dose, and the indication for sedation by an endoscopist or an anaesthetist can be varied depending on the results obtained. It must be acknowledged that the limit between deep sedation and general anaesthesia is very subtle; therefore the concepts of deep and superficial sedation and anaesthesia must be well understood [10].

In our experience, sedation by a team trained in deep sedation and advanced resuscitation manoeuvres allows the appropriate level of sedation to be maintained with the lowest possible risk and is therefore a valid alternative to sedation by a trained anaesthetist.

Optimisation of the colonic cleansing quality and sedation by the endoscopist improve the efficiency of the procedure by reducing the need to repeat inadequately prepared colonoscopies, which results in greater lesion detection, and eliminates the need for a pre-operative anaesthesia consultation.

Likewise, the establishment of a fast CRC diagnosis, follow-up and treatment loop results in a better performance of population-based screening programs [29]. Our program included a commitment to perform a colonoscopy within less than one month in the event of a positive iFOBT, with a similar time frame for radiological and surgical staging.

More than $90 \%$ of CRCs detected at an early stage can be cured. Whereas diagnosis at a more advanced stage, which occurs in more than $40 \%$ of cases, results in higher average diagnosis and treatment costs. CRC is preventable. The implementation of population-based screening programs reduces the incidence and mortality [1][3]. Early diagnosis and cancer prevention due to screening imply a saving of at least $40 \%$ compared to the direct costs resulting from CRC treatment [30].

As demonstrated by various studies and systematic reviews, any type of CRC screening (FOBT, sigmoidoscopy or colonoscopy) is cost-effective when compared to no screening. The most cost-effective screening strategy in Spain is an annual iFOBT [2] [31].

In order for a CRC screening program to be effective, it is important to be able to rely on a database that includes the entire target population, good communication, coordination between different regions, guaranteed health-care continuity and follow-up of patients with lesions, as well as a quality plan. These requirements are fulfilled in our screening program. It is also vital to raise awareness in the population and the health regions concerned [1] [31] and [32].

\section{Conclusion}

Although it requires an initial investment, the CRC screening program is highly effective. Dividing the dose of the colonic cleansing preparation and reducing the time interval between the second dose and the colonoscopy result in a high percentage of adequate preparation and lesion detection. Sedation by trained endoscopists is safe and effective. An immunological faecal occult blood test-based screening program is feasible and allows malignant and precancerous lesions to be efficiently detected and treated, thereby decreasing the incidence of colorectal cancer. 


\section{Acknowledgements}

We would like to thank Isabel Portillo and Isabel Idígoras from the ACBC CRC screening program coordination centre for their help with collecting data for the Basque Country, and Dra. Laura Gomez Irwin and Bioef for funding the translation of this manuscript into English.

\section{Conflict of Interest}

The authors report no conflicts of interest.

\section{References}

[1] Segnan, N., Patnick, J. and Von Karsa, L. (2010) European Guidelines for Quality Assurance in Colorectal Cancer Screening and Diagnosis. International Agency for Research on Cancer. European Commision, Belgium.

[2] Morillas, J.M., Castells, A., Oriol, I., Pastor, A., Pérez-Segura, P., Echevarría, J.M., et al. (2012) Alianza para la prevención del cáncer de colon en España: Un compromiso cívico con la sociedad. Gastroenterología y Hepatología, 35, 109-128. http://dx.doi.org/10.1016/j.gastrohep.2012.01.002

[3] Jover, R. (2011) Guía de práctica clínica de calidad en la colonoscopia de cribado de cáncer colorrectal. Grupo de trabajo AEG-SEED, EDIMSA S.A.

[4] Ministerio de Sanidad y Política Social (2010) Estrategia en Cáncer del Sistema Nacional de Salud. Actualización aprobada por el Consejo Interterritorial del Sistema Nacional de Salud, el 29 de octubre de 2009.

[5] Rex, D.K. (2007) Optimizing Bowel Preparation for Colonoscopy. Gastroenterology \& Endoscopy News Special Edition, 7-11.

[6] Cohen, L.B., Kastenberg, D.M., Mount, D.B. and Safdi, A.V. (2009) Current Issues in Optimal Bowel Preparation. Gastroenterology \& Hepatology, 5, 1-11.

[7] Ojeda, R. (2011) Capítulo 4. Anestesiología básica. In: de la Morena Madrigal, E. and Acosta, G.C., Eds., Sedación en endoscopia digestiva, Medicos, S.A. EDIMSA, Madrid, 27-40.

[8] An Update Report by the American Society of Anesthesiologists Committee on Sandards and Practice Parameters (2011) Practice Guidelines for Preoperative Fasting and the Use of Phamarcologic Agents to Reduce the Risk of Pulmonary Aspiration: Applications to Healthy Patients Undergoing Elective Procedures. Anesthesiology, 114, 495-511. http://dx.doi.org/10.1097/ALN.0b013e3181fcbfd9

[9] Lai, E.J., Calderwood, A.H., Doros, G., Fix, O.K. and Jacobson, B.C. (2009) The Boston Bowel Preparation Scale: A Valid and Reliable Instrument for Colonoscopy-Oriented Research. Gastrointestinal Endoscopy, 69, 620-625. http://dx.doi.org/10.1016/j.gie.2008.05.057

[10] Simon, M.A., Bordas, J.M., Campo, R., González-Huix, F., Igea, F., Monés, J. and en representacion del grupo de endoscopia de la asociacion española de gastroenterologia (2006) Documento de consenso de la asociacion española de gastroenterología sobre sedoanalgesia en la endoscopia digestiva. Gastroenterología y Hepatología, 29, 131-149. http://dx.doi.org/10.1157/13085143

[11] Repici, A., Pagano, N., Hassan, C., Carlino, A., Rando, G., Strangio, G., et al. (2011) Balanced Propofol Sedation Administered by Nonanesthesiologist: The First Italian Experience. World Journal of Gastroenterology, 17, 3818-3823. http://dx.doi.org/10.3748/wjg.v17.i33.3818

[12] Jass, J.R. and Sabin, L.H. (1989) Histological Typing of Intestinal Tumours. 2nd Edition, Springer-Verlag, New York. http://dx.doi.org/10.1007/978-3-642-83693-0

[13] Grupo de trabajo de la guía de práctica clínica de prevención del cáncer colorrectal. Actualización del 2009. Guía de práctica clínica. Barcelona: Asociación Española de Gastroenterología, Sociedad Española de Medicina de Familia y Comunitaria, y Centro Cochrane Iberoamericano; 2009. Programa de elaboración de Guías de Práctica Clínica en Enfermedades Digestivas, desde la Atención Primaria a la Especializada: 4.

[14] Sabin, L.H. and Wittekind, C. (2002) TNM Classification of Malignant Tumours. 6th Edition, Wiley-Liss, New York.

[15] Bakker, C.K., Jonkers, D., Smits, K., Mesters, I., Masclee, A. and Stockbrügger, R. (2011) Participation in Colorectal Cancer Screening Trials after First-Time Invitation: A Systematic Review. Endoscopy, 43, 1059-1086. http://dx.doi.org/10.1055/s-0031-1291430

[16] Málaga López, A., Salas Trejo, D., Sala Felis, T., Ponce Romero, M., Goicoechea Sáez, M., Andrés Martínez, M., et al. (2012) Programa de cribado de cáncer colorrectal de la comunidad valenciana. Resultados de la primera ronda: 2005-2008. Revista Española de Salud Pública, 84, 731-743.

[17] Navarro, M., Peris, M., Binefa, G., Nogueira, J.M., Miquel, J.M., Espinás, J.A., Borrás, J.M. and el Grupo Catalán del 
Programa Piloto en Cribado de Cáncer Colorrectal (2008) Hallazgos colonoscópicos del estudio piloto de cribado de cáncer colorrectal realizado en Cataluña. Revista Española de Enfermedades Digestivas, 100, 343-348. http://dx.doi.org/10.4321/S1130-01082008000600006

[18] Morán Sánchez, S., Torella, E., Esteban Delgado, P., Baños Madrid, R., García, A., Ono, A., Pérez Cuadrado, E., Parra, P., Cruzado Quevedo, J., Pérez Riquelme, F. and Carballo Álvarez, F. (2009) Valoración de la calidad en la práctica de la colonoscopia. Revista Española de Enfermedades Digestivas, 101, 107-116.

[19] Van Rossum, L.G.M., Van Rijn, A.F., Laheij, R.J., Van Oijen, M.G., Fockens, P., Van Krieken, H.H., Verbeekx, A.L., Jansen, J.B. and Dekkerx, E. (2008) Radom Comparison of Guaiac and Immunochemical Fecal Occult Blood Tests for Colorectal Cancer in a Screening Population. Gastroenterology, 135, 82-90. http://dx.doi.org/10.1053/j.gastro.2008.03.040

[20] Navarro, M., Bienfa, G., Blanco, I., Guardiola, J., Rodríguez-Moranta, F., Peris, M. and the Catalan Colorectal Cancer Screening Pilot Programme Group (2009) Colorectal Cancer Screening: Strategies to Select Populations with Moderate Risk for Disease. Revista Española de Enfermedades Digestivas, 101, 855-860. http://dx.doi.org/10.4321/S1130-01082009001200005

[21] Portillo, I., Idígoras, I., Ojembarrena, E., Arana, E., Hurtado, J.L., Basurko, R., Tapiaa, M. and Luz Peña, M. (2013) Lesiones detectadas en el programa de cribado de cáncer colorrectal en el País Vasco primera ronda 2009-2011. Gastroenterología y Hepatología, 36, 301-308. http://dx.doi.org/10.1016/j.gastrohep.2013.02.004

[22] Hawes, R.H., Lowry, A. and Deziel, D. (2006) A Consensus Document on Bowel Preparation before Colonoscopy: Prepared by a Task Force from the American Society of Colon and Rectal Surgeons, the American Society for Gastrointestinal Endoscopy, and the Society of American Gastrointestinal and Endoscopic Surgeons. Gastrointestinal Endoscopy, 63, 894-909. http://dx.doi.org/10.1016/j.gie.2006.03.919

[23] González, F., Llado, H., Figa Francesh, M. and Huertas Nadal, C. (2010) Criterios de calidad que deben exigirse en la indicación y en la realización de la colonoscopia. Gastroenterología y Hepatología, 33, 33-42. http://dx.doi.org/10.1016/j.gastrohep.2009.02.014

[24] Siddiqui, A.A., Yang, K., Spechler, S.J., Cryer, B., Davila, R., Cipher, D. and Harford, W.V. (2009) Duration of the Interval between the Completion of Bowel Preparation and the Start of Colonoscopy Predicts Bowel-Preparation Quality. Gastrointestinal Endoscopy, 69, 700-706.

[25] Parra-Blanco, A., Nicolás-pérez, D., Gimeno-García, A., Grosso, B., Jiménez, A., Ortega, J. and Quintero, E. (2006) The Timing of Bowel Preparation before Colonoscopy Determines the Quality of Cleansing, and Is a Significant Factor Contributing to the Detection of Flat Lesions: A Randomized Study. World Journal of Gastroenterology, 12, 61616166.

[26] Seo, E.H., Kim, T.O., Park, M.J., Joo, H.R., Heo, N.Y., Park, J., Park, S.H., Yang, S.Y. and Moon, Y.S. (2011) Optimal Preparation-to-Colonoscopy Interval in Split-Dose PEG Bowel Preparation Determines Satisfactory Bowel Preparation Quality: An Observational Prospective Study. Gastrointestinal Endoscopy, 20, 1-8.

[27] Rodriguez De Miguel, C., Serradesanferm, A., Del Manzano, S., Cárdenas, A., Fernández-Esparrach, G., et al. (2012) La hora de ingesta del polietilenglicol es un factor clave en la tolerancia y eficacia de la preparación del colon en individuos de un programa poblacional de cribado de cáncer colorrectal. Gastroenterología y Hepatología, 35, $236-242$. http://dx.doi.org/10.1016/j.gastrohep.2012.01.012

[28] Vargo, J.J., Cohen, L.B., Rex, D.K. and Kwo, P.Y. (2009) Position Statement: Nonanesthesiologist Administration of Propofol for GI Endoscopy. Gastroenterology, 137, 2161-2167. http://dx.doi.org/10.1053/j.gastro.2009.09.050

[29] Vallverdú Cartié, H., Comajuncosas Camp, J., Orbeal Sáenz, R.A., López Negre, J.L., Gris Garriga, P.J., Jimeno Fraile, J., Bosch, J.H., Pradell, C.S., Alsina, S.T., Urgellés Bosch, J. and Parés, D. (2011) Resultados de la implementación del circuito de diagnóstico rápido de cáncer colorrectal. Revista Española de Enfermedades Digestivas, 103, $402-407$. http://dx.doi.org/10.4321/S1130-01082011000800003

[30] Carballo, F. and Muñoz-Navas, M. (2012) Prevenir o curar en época de crisis: A propósito del cribado de cáncer de colon y recto. Revista Española de Enfermedades Digestivas, 104, 537-545. http://dx.doi.org/10.4321/S1130-01082012001000006

[31] Castells, A., Marzo-Castillejo, M., Mascort, J.J., Amador, F.J., Andreu, M., Bellas, B., et al. (2009) Prevención del cáncer colorrectal. Actualización 2009. Gastroenterología y Hepatología, 32, 717.et-717.e58.

[32] Grau, J., Serradesanferm, A., Polbach, S., García-Basteiro, A.L., Trilla, A. and Castells, A. (2010) Programas de cribado del cáncer colorrectal en la población de riesgo medio en la Unión Europea y España. Gastroenterología y Hepatología, 33, 111-118. http://dx.doi.org/10.1016/j.gastrohep.2009.03.007 